\title{
Clinicopathologic Features and Outcome Predictors of Leptospira interrogans Australis Serogroup Infection in Dogs: A Retrospective Study of 20 Cases (2001-2004)
}

\author{
Cinzia Mastrorilli, Francesco Dondi, Chiara Agnoli, Maria Elena Turba, Enrico Vezzali, \\ and Fabio Gentilini
}

\begin{abstract}
Background and Hypothesis: We retrospectively evaluated the clinicopathologic findings and outcome predictors in dogs with Leptospira interrogans Australis serogroup infections.

Animals and Methods: The medical records of 159 dogs that had a leptospiral microscopic agglutination test (MAT) performed between 2001 and 2004 were reviewed.

Results: Twenty dogs met serologic criteria for either symptomatic (16 dogs) or asymptomatic (4 dogs) infection caused by Leptospira interrogans Australis serogroup. Seven of 16 symptomatic dogs died or were euthanized and 9/16 recovered. Systemic inflammatory response syndrome (SIRS) was observed in 9/16 dogs. The presence of SIRS did not affect prognosis $(P=.357)$. C-reactive protein $(\mathrm{CRP})$ and haptoglobin $(\mathrm{Hpt})$ concentrations were altered in all symptomatic dogs, but results did not differ significantly between survivors and nonsurvivors $(P=.08$ and $P=.055$, respectively). Conversely, the CRP to $\mathrm{Hpt}$ ratio $(\mathrm{CRP} / \mathrm{Hpt})$ was significantly increased in nonsurvivors. Disseminated intravascular coagulation (DIC) was diagnosed in $7 / 16$ dogs. DIC did not significantly affect outcome $(P=.126)$. Multiple organ involvement was present with renal failure in $16 / 16$, liver damage in $12 / 16$, cardiac damage in $11 / 16$, and muscular damage in $8 / 16$ dogs.

Conclusions and Clinical Importance: Among the evaluated clinicopathologic biomarkers, serum albumin, cardiac troponin I, CRP/Hpt, urinary albumin, and urinary total protein to creatinine ratio were found to predict outcome and warrant evaluation in larger prospective studies.
\end{abstract}

Key words: Cardiac troponin I; Coagulation; Prognosis; Systemic inflammatory response syndrome (SIRS); Urinary albumin.

$\mathbf{L}$ eptospirosis is a reemerging zoonotic disease and apparently new serovars have been recognized in dogs during the last 10 years. ${ }^{1-4}$ Clinical signs and clinicopathologic findings of emerging leptospirosis are quite variable, ranging from subclinical infections to severe illness with multiple organ involvement..$^{5-8}$ Acute renal failure remains the most common finding in infected dogs, but other organs and tissues such as muscle, liver, and blood vessels may be involved in unpredictable combinations. ${ }^{2,3,8}$ Pulmonary, cardiac, and central nervous system (CNS) involvement, increasingly reported in human patients, is not well defined in dogs with leptospirosis. ${ }^{6,8-11}$ Currently, a diagnosis of leptospirosis is based on a combination of clinical signs, clinicopathologic findings, and the micro-

From the Veterinary Clinical Department (Mastrorilli, Dondi, Agnoli, Gentilini), the Department of Veterinary Morphophysiology and Animal Production (Turba), and the Department of Veterinary Public Health and Animal Pathology (Vezzali), Faculty of Veterinary Medicine, Alma Mater Studiorum-University of Bologna, Ozzano dell'Emilia, Bologna, Italy. Preliminary results of this study were presented as a poster communication at the 13th European College of Veterinary Internal Medicine-Companion Animals (ECVIM-CA) Congress, 4-6 September 2003, Uppsala, Sweden.

Reprint requests: Gentilini Fabio, DVM, Dipartimento Clinico Veterinario, Università di Bologna, via Tolara di Sopra 50, 40064, Ozzano dell'Emilia, Bologna, Italia; e-mail:fgentilini@vet.unibo.it.

Submitted August 17, 2005; Revised January 17, 2006; April 14, 2006; May 16, 2006; June 16, 2006; August 17, 2006; Accepted September 18, 2006.

This study was performed at the Veterinary Clinical Department, Faculty of Veterinary Medicine, Alma Mater Studiorum-Università di Bologna, Ozzano dell'Emilia, Italy.

Copyright $(2007$ by the American College of Veterinary Internal Medicine

0891-6640/07/2101-0001/\$3.00/0 scopic agglutination test (MAT). MAT is still considered the "gold standard" assay ${ }^{2,46,10}$ despite recent evidence questioning its reliability. ${ }^{1,12}$ Survival rates ranging from $53 \%$ to $86 \%$ have been reported. Of the few studies that have investigated outcome predictors, only renal markers such as creatinine were shown to be linked with outcome. ${ }^{2,6,8}$

Although progress has been made in the basic research of leptospirosis, the mechanism of pathogenesis remains to be elucidated. ${ }^{1,9,11}$ Experimental studies have shown that both leptospiral virulence (ie, expression of strain or serovar specific toxins) and host immune response account for the severity and clinical features of the disease..$^{3,5,11,13}$ In particular, there is evidence that many of the complications of spirochetal diseases are caused by the host inflammatory response involving the cytokine network. ${ }^{11,14}$ Cytokines trigger the systemic inflammatory response syndrome (SIRS), activate coagulation, and impair fibrinolysis, eventually leading to or worsening multiple organ dysfunction syndrome (MODS). ${ }^{14,15}$ In human medicine, SIRS and MODS criteria have been refined in recent years, greatly improving their predictive usefulness for many diseases. ${ }^{15}$ Conversely, the criteria adapted for MODS and SIRS in veterinary medicine are not widely accepted and need to be further evaluated in the clinical setting. ${ }^{15}$

The purpose of this study was to describe the clinicopathologic features of infections caused by Leptospira interrogans Australis serogroup (LIAS) with emphasis on the occurrence of SIRS, disseminated intravascular coagulation (DIC), and multiple organ involvement. In addition, the effect of these syndromes and of newly validated biomarkers such as cardiac troponin I (cTnI), C-reactive protein (CRP), haptoglobin $(\mathrm{Hpt})$, and urinary albumin on outcome also was evaluated. 


\section{Materials and Methods}

The medical records of 159 dogs that had been admitted to the University of Bologna Veterinary Teaching Hospital (UBVTH) between September 2001 and September 2004 and that had had leptospiral MAT performed were reviewed. One of the following criteria was required for inclusion in the study: (1) clinical signs of leptospirosis and (a) a titer against Australis serogroup $\geq 1: 800$, (b) a 4-fold increase in titer in paired sera against Australis serogroup, or (c) a titer $\geq 1: 200$ against Australis serogroup without reactivity to other serogroups, or (2) apparently normal health status with (a) a titer $\geq 1: 3200$ on admission, or (b) a 4-fold increase in titer in paired sera against Australis serogroup. Dogs with negative MAT, including polymerase chain reaction (PCR)-positive dogs without molecular typing $(\mathrm{n}=2)$ and dogs with positive MAT with predominant titer against serogroups other than Australis $(\mathrm{n}=4)$ were excluded. One other dog with a predominant titer against Australis serogroup and concurrent disease (ie, spinal cord trauma) also was excluded.

A computerized database was used to search medical records based on the inclusion criteria. Clinical information was recorded including MAT results, signalment, history, physical examination, clinicopathologic data, results of ancillary tests (radiography, ultrasonography, and electrocardiography), urine culture results, pathologic findings, treatments, and outcome. The MAT performed at the Animals National Leptospirosis Referral Laboratory ${ }^{\mathrm{a}}$ was able to determine titers against 8 serogroups: Australis, Ballum, Canicola, Grippotyphosa, Icterohemorragiae, Pomona, Sejroe, and Tarassovi. Positive sera with a screening dilution of 1:100 were titered until an end point of 1:6400. If performed, convalescent titers also were recorded. The following initial hematologic data were collected on admission: $\mathrm{CBC}$; serum biochemical profile including cTnI, CRP, and $\mathrm{Hpt}$; and a coagulation panel including platelet count, prothrombin time (PT), activated partial thromboplastin time (aPTT), fibrinogen, antithrombin (AT), and d-dimer. The CRP to Hpt ratio (CRP/Hpt) was calculated.

Serum $\mathrm{CTnI}^{\mathrm{b}}$ and $\mathrm{Hpt}^{\mathrm{c}}$ concentrations were determined by assays designed for humans and validated for dogs in the Veterinary Clinical Department (VCD) laboratory according to standard validation protocols which included intra-assay and interassay coefficients of variation $<10 \%$ and linearity and recovery between 80 and $120 \%$. CRP was measured by a canine-specific enzyme-linked immunosorbent assay (ELISA). ${ }^{d}$ D-dimer ${ }^{\mathrm{e}}$ was measured by a latex-immunoturbidimetric assay. The following markers were retrospectively assayed on stored frozen samples: cTnI $(\mathrm{n}=1), \operatorname{Hpt}(\mathrm{n}=7)$, and CRP $(\mathrm{n}=20)$ on serum; and AT, fibrinogen, and d-dimer $(n=5)$ on citrated plasma.

Urinalysis (performed on admission) results including dipstick, urine specific gravity (USG), sediment evaluation, urinary total protein to creatinine ratio (UPC), and urinary albumin to creatinine ratio (UAC) were recorded. After testing, remaining urine samples were frozen within 2 hours of collection at $-20^{\circ} \mathrm{C}$. Urine samples were collected by cystocentesis. The urinary albumin concentration was determined by an automated immunoturbidimetric assay ${ }^{\mathrm{g}}$ designed for humans and validated for dogs in the VCD laboratory. ${ }^{16}$ A UAC $>0.05$ was considered abnormal. ${ }^{17}$ Proteinuria was qualitatively evaluated by sodium dodecyl sulphate-polyacrylamide gel electrophoresis (SDS-PAGE) ${ }^{\mathrm{h}}$ on frozen samples. Five micrograms of urinary protein were loaded for each sample. Bands in the range of 3 to $300 \mathrm{kDa}$ were identified. Qualitative proteinuria was classified by visual inspection according to previous reports in the human and canine medical literature ${ }^{18,19}$ as follows: (1) physiologic - absence of any or presence of only faint albumin bands; (2) glomerular - presence of albumin bands associated with high molecular weight proteins (molecular weight $>69 \mathrm{kDa}$ ); (3) tubular - presence of bands with molecular weight lower than albumin (low molecular weight proteins); and (4) mixed - presence of both glomerular and tubular bands. Tubular proteinuria was further classified as " $>23 \mathrm{kDa}$ " and " $<23 \mathrm{kDa}$ " based on the localization of the band closest to the cathode.

Based on clinical history and physical examination, dogs were classified as symptomatic or asymptomatic. The symptomatic dogs were retrospectively categorized as SIRS positive if at least 2 of 4 SIRS criteria (heart rate $>120 \mathrm{bpm}$, respiratory rate $>40 \mathrm{bpm}$, rectal temperature $>40^{\circ} \mathrm{C}$ or $<38^{\circ} \mathrm{C}$, and leukogram $>18,000$ white blood cells $/ \mu \mathrm{L}$ or $<5000 / \mu \mathrm{L}$ ) were present at the time of admission, as previously reported..$^{20,21}$ Dogs were categorized as DIC positive if at least 3 of the following criteria were present at the time of admission: decreased platelet count, prolonged PT, prolonged aPTT, reduction of fibrinogen concentration, reduction of AT activity, and increased d-dimer concentration. ${ }^{15}$ Increases in the following biochemical variables were considered evidence of specific organ and tissue damage: serum creatinine concentration (kidney), cTnI concentration (heart), and creatine kinase (CK) activity (muscle). Increases in at least 3 of the following variables were considered evidence of hepatic damage: alanine aminotransferase (ALT) activity, aspartate aminotransferase (AST) activity, alkaline phosphatase (ALP) activity, gamma-glutamyltransferase (GGT) activity, and bilirubin concentration. Dogs were categorized based on the number of organs and tissues involved. Symptomatic dogs were further classified according to outcome as survivors or nonsurvivors.

\section{Statistical Analyses}

All data were evaluated by standard descriptive statistics. Quantitative variables between survivors and nonsurvivors were compared by the Mann-Whitney $U$ test. Fisher exact test was used to compare the effect of SIRS, DIC, and organ and tissue damage on outcome. The proportion of dogs with urinary casts between groups was compared by Fisher exact test. Linear regression analysis was performed and the Pearson $R$ correlation coefficient was calculated after log transformation of serum $\mathrm{cTnI}$ and urea concentration data. A $P$ value of $<.05$ was considered statistically significant.

\section{Results}

Twenty dogs met the inclusion criteria $(1 \mathrm{a}, 9 / 20 ; 1 \mathrm{~b}$, $1 / 20 ; 1 c, 6 / 20 ; 2 a, 3 / 20 ; 2 b, 1 / 20$ ). Sixteen of $20 \mathrm{dogs}$ showed clinical and clinicopathologic findings consistent with leptospirosis. The remaining 4 dogs were asymptomatic but shared a household with other infected dogs in this study. Serologic results on admission are shown in Figure 1.

MAT was performed on convalescent sera in 11/20 dogs (median of 27 days after admission) and yielded a positive titer against Australis serogroup as the only serogroup or in association with other serogroups. In the latter case, the titer against Australis serogroup was always predominant. Convalescent titers increased in $2 / 11$, decreased in $5 / 11$, and were unchanged (end-point dilution, 1:6400) in 4/11 dogs.

Overall, the median age of the dogs was 8.5 years (range, 3-13 years). Fourteen of $20 \mathrm{dogs}$ were sexually intact males, 4/20 were intact females, and 2/20 were spayed females. Eighteen of 20 patients had frequented rural environments and, in particular, 9/20 dogs were 


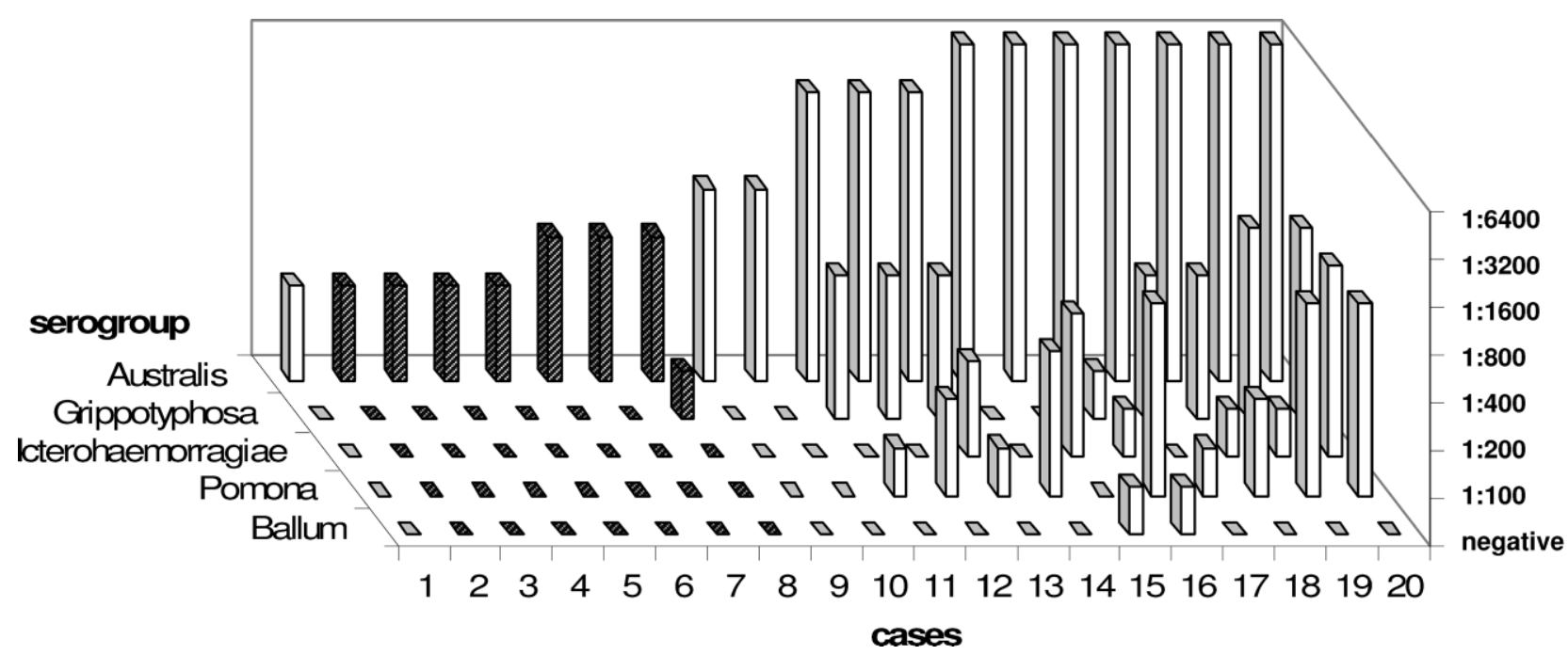

Fig 1. Microscopic agglutination test (MAT) results upon admission of the 20 dogs included in the study. The MAT, performed against Canicola, Sejroe, and Tarassovi serogroups, always yielded negative results. Open bars $=$ survivors; striped bars $=$ nonsurvivors.

habitually used for hunting. Two of 20 dogs lived in fenced yards. Fifteen of 20 subjects had been vaccinated with a polyvalent vaccine that included Canicola and Icterohemorragiae serovars within the past year but not in the past 3 months, whereas $5 / 20$ dogs had not been regularly vaccinated.

In the symptomatic dogs (16/20), attending clinicians defined the onset as acute (sudden onset of illness in a previously healthy dog) in 15/16 dogs and as subacute (onset of illness with previous symptoms of polyuria and polydipsia within the previous 10 days) in 1/16 dogs. The median length of time between the onset of clinical signs and UBVTH admission was 6.5 days. The clinical findings on admission are presented in Table 1 .

Mild, nonregenerative anemia was present on the CBC in 6/16 dogs and the hematocrit was increased in 2/ 16 dogs. Ten of 16 dogs had neutrophilic leukocytosis

Table 1. Clinical signs in symptomatic dogs $(n=16)$ upon admission.

\begin{tabular}{lc}
\hline \multicolumn{1}{c}{ Signs } & No. of Dogs \\
\hline Lethargy & 14 \\
Vomiting & 13 \\
Anorexia & 11 \\
Recumbency & 7 \\
Oliguria or Anuria & 7 \\
Tachycardia (heart rate $>120 \mathrm{bpm}$ ) & 7 \\
Polypnea/Dyspnea (respiratory rate $>40 \mathrm{bpm}$ ) & 7 \\
Abdominal pain & 6 \\
Diarrhea & 6 \\
Hypothermia (rectal temperature $<38^{\circ} \mathrm{C}$ ) & 6 \\
Hyperthermia (rectal temperature $\left.\geq 39.5^{\circ} \mathrm{C}\right)$ & 3 \\
Arrhythmias & 3 \\
Icterus & 2 \\
Bleeding & \\
Hemorrhagic nasal discharge & 3 \\
Melena & 3 \\
Petechiae & 2 \\
Macroscopic hematuria & 1 \\
\hline
\end{tabular}

and $1 / 16$ dogs had leukopenia. Nine of 16 dogs were classified as SIRS-positive and 7/16 dogs as SIRSnegative. Serum CRP concentration was increased in all 16 dogs and Hpt concentration was increased in 15/16 dogs and below the reference range in $1 / 16$ dogs. DIC was diagnosed in 7/16 dogs. Complete coagulation panel results are presented in Table 2.

Biochemical data (Table 3) was consistent with multiple organ damage with simultaneous involvement of liver, muscle, and heart in variable association with a consistent presence of azotemia and hyperphosphatemia. Two dogs of 16 had a single organ involved (kidney). The organ combinations were kidney/heart (2/ 16), kidney/liver (2/16), kidney/heart/liver (2/16), and kidney/muscle/liver (1/16), kidney/heart/muscle/liver (7/ 16). cTnI concentrations were increased in $11 / 16$ dogs and ranged from 0.4 to $60.1 \mathrm{ng} / \mathrm{mL}$. cTnI concentration was significantly correlated with $\mathrm{CK}(R=0.669, P=$ $.005)$ and AST $(R=0.749, P=.001)$ but not with urea $(R=0.407, P=.118)$.

Urinalysis (including UAC and UPC) and urine SDSPAGE results were available for 15/16 and 13/16 symptomatic dogs, respectively. Complete urinalysis data are presented in Table 4. Bacterial growth was not identified in any of the 10 urine cultures performed. One dog had bacteriuria on sediment examination, but the urine was not cultured. In the same dog, SDS-PAGE was not performed, and UPC as well as UAC results were excluded from the statistical analysis because of evidence of urinary tract infection. UPC and UAC were increased in 14/14 symptomatic dogs tested (UPC: median, 3.8; range, 0.9-19.7; reference range, 0.0-0.4; UAC: median, 1.43; range, 0.06-7.31; reference range, $0.0-0.05)$.

Abdominal ultrasound examinations were performed in 12/16 symptomatic dogs and showed hyperechogenicity of the renal cortex in 1/12 dogs, changes suggestive of cholangiohepatitis in 1/12 dogs, and mild peritoneal effusion in 3/12 dogs. Two dogs had thoracic radiographs taken, and a disseminated pulmonary interstitial 
Table 2. Coagulation panel results in symptomatic $\operatorname{dogs}(\mathrm{n}=16)$ upon admission.

\begin{tabular}{lcccc}
\hline \multicolumn{1}{c}{ Variable } & Median $(\mathrm{n}=16)$ & Range $(\mathrm{n}=16)$ & Reference Range & No. of Abnormal Cases $(\%)$ \\
\hline Antithrombin $(\%)$ & 79 & $31-109$ & $105-166$ & $15(93.75)$ \\
Fibrinogen $(\mathrm{g} / \mathrm{L})$ & 4.59 & $2.21-7.57$ & $1.45-3.85$ & $12(75)$ \\
D-dimer $(\mu \mathrm{g} / \mathrm{mL})$ & 0.42 & $0.06-2.71$ & $0-0.26$ & $10(62.5)$ \\
Platelet count $\left(\times 10^{6} / \mu \mathrm{L}\right)$ & 178 & $37-543$ & $160-500$ & $8(50)$ \\
PT (seconds) & 7.5 & $6.4-8.8$ & $5.0-7.5$ & $1(6.25)$ \\
aPTT (seconds) & 13.6 & $9.7-29.1$ & $8.0-16.5$ & $1(6.25)$ \\
\hline
\end{tabular}

PT, prothrombin time; aPTT, activated partial thromboplastin time.

pattern was detected in 1 dog. An electrocardiogram (ECG) was performed in 3 dogs that had rhythm disturbances on physical examination at admission. Ventricular tachyarrhythmias were identified in all 3 dogs.

Renal biopsies were performed in 8/16 symptomatic dogs and a postmortem examination was performed in $1 / 16$ dogs. One of 8 biopsy samples was considered inadequate for diagnostic purposes. Tubulointerstitial nephritis was found in 8 specimens and included evidence of lymphoplasmocytic infiltration and mild interstitial fibrosis in $7 / 8$ dogs and acute damage (ie, tubular regenerative pattern) in $1 / 8$ dogs. Tubules showed different grades of involvement (ie, epithelial hyperplasia to tubular ectasia) combined with degenerative lesions (eosinophilic or periodic acid-Schiff (PAS)positive casts). Moreover, glomerular abnormalities (ranging from glomerular hyperemia and mesangial matrix expansion to marked fibrinoid change, necrosis, or sclerosis) were found in $7 / 8$ dogs. One dog had glomerular amyloidosis associated with nonamyloidotic tubulointerstitial inflammatory infiltration.

All symptomatic dogs were treated with ampicillin or amoxicillin in addition to other supportive treatments until death or recovery followed by doxycycline for 2 weeks in those that recovered. One dog was treated with intermittent hemodialysis.

Nine of 16 symptomatic dogs recovered, 5/16 died, and $2 / 16$ were euthanized due to progressive worsening of their condition. The length of time between the onset of clinical signs and admission to UBVTH was not statistically different between survivors and nonsurvivors (median, 5 and 7 days, respectively; $P=.89$ ). Five of 7 nonsurvivors and 4/9 survivors were SIRS-positive. Being SIRS-positive did not have a significant impact on outcome $(P=.357)$. Five of 7 nonsurvivors and $2 / 9$

Table 3. Biochemistry results including cardiac Troponin I, C-reactive protein, and haptoglobin in symptomatic dogs $(\mathrm{n}=16)$ on admission.

\begin{tabular}{lcccc}
\hline \multicolumn{1}{c}{ Variable } & Median $(\mathrm{n}=16)$ & Range $(\mathrm{n}=16)$ & Reference Range & No. of Abnormal Cases $(\%)$ \\
\hline Creatinine $(\mathrm{mg} / \mathrm{dL})$ & 7.52 & $1.77-20.77$ & $0.65-1.35$ & $16(100)$ \\
Urea $(\mathrm{mg} / \mathrm{dL})$ & 370 & $91-859$ & $18-55$ & $16(100)$ \\
CRP $(\mathrm{mg} / \mathrm{dL})$ & 5.90 & $0.89-13.65$ & $0-0.5$ & $16(100)$ \\
Haptoglobin $(\mathrm{mg} / \mathrm{dL})$ & 216 & $12-501$ & $20-140$ & $16(100)$ \\
Phosphorus $(\mathrm{mg} / \mathrm{dL})$ & 15.0 & $4.7-35$ & $2.6-4.9$ & $15(93.75)$ \\
Albumin $(\mathrm{g} / \mathrm{dL})$ & 2.57 & $0.74-3.27$ & $2.80-3.70$ & $12(75)$ \\
cTnI $(\mathrm{ng} / \mathrm{mL})$ & 6.3 & $0.1-60.1$ & $<0.3$ & $11(68.75)$ \\
AST $(\mathrm{U} / \mathrm{L})$ & 71 & $24-815$ & $20-42$ & $11(68.75)$ \\
ALT $(\mathrm{U} / \mathrm{L})$ & 84 & $9-178$ & $20-55$ & $10(68.75)$ \\
ALP $(\mathrm{U} / \mathrm{L})$ & 265 & $40-1123$ & $92.5)$ \\
Total bilirubin $(\mathrm{mg} / \mathrm{dL})$ & 0.38 & $0.12-9.83$ & $0.07-0.34$ & $9(56.25)$ \\
Amylase $(\mathrm{U} / \mathrm{L})$ & 1139 & $429-3974$ & $340-980$ & $7(43.75)$ \\
CK $(\mathrm{U} / \mathrm{L})$ & 257 & $53-17,333$ & $50-290$ & $7(43.75)$ \\
Glucose $(\mathrm{mg} / \mathrm{dL})$ & 105 & $49-292$ & $70-125$ & $6(37.5)$ \\
Total protein $(\mathrm{g} / \mathrm{dL})$ & 6.26 & $3.50-8.90$ & $5.60-7.90$ & $5(31.25)$ \\
Bile acid $(\mu \mathrm{mol} / \mathrm{L})$ & 4.5 & $2.1-13.7$ & $2.5-9$ & $5(31.25)$ \\
Cholesterol $(\mathrm{mg} / \mathrm{dL})$ & 293 & $164-747$ & $140-350$ & $5(31.25)$ \\
Tryglyceride $(\mathrm{mg} / \mathrm{dL})$ & 76 & $24-538$ & $30-120$ & $5(31.25)$ \\
Calcium $(\mathrm{mg} / \mathrm{dL})$ & 10.9 & $7-13.4$ & $9-11.8$ & $5(31.25)$ \\
Potassium $(\mathrm{mEq} / \mathrm{L})$ & 4.7 & $3.9-9.2$ & $3.9-5.3$ & $4(25)$ \\
Chloride $(\mathrm{mEq} / \mathrm{L})$ & 104 & $73-112$ & $108-118$ & $4(25)$ \\
GGT $(\mathrm{U} / \mathrm{L})$ & 4.6 & $0.1-12.9$ & $0-5.8$ & $4(25)$ \\
Total iron $(\mu \mathrm{g} / \mathrm{dL})$ & 73 & $24-198$ & $50-230$ & $3(18.75)$ \\
Sodium $(\mathrm{mEq} / \mathrm{L})$ & 146 & $125-150$ & $143-154$ & $3(18.75)$ \\
Lipase $(\mathrm{U} / \mathrm{L})$ & 294 & $88-2750$ & $70-700$ & \\
\hline
\end{tabular}

CRP, C-reactive protein; cTnI, cardiac Troponin I; AST, aspartate aminotransferase; ALT, alanine aminotransferase; ALP, alkaline phosphatase; CK, creatine kinase; GGT, gamma-glutamyltransferase. 
Table 4. Urinary findings in symptomatic and asymptomatic dogs upon admission.

\begin{tabular}{|c|c|c|}
\hline \multirow[b]{2}{*}{ Parameter } & \multicolumn{2}{|c|}{ No. of Cases in } \\
\hline & $\begin{array}{c}\text { Symptomatic } \\
\text { Dogs }\end{array}$ & $\begin{array}{c}\text { Asymptomatic } \\
\text { Dogs }\end{array}$ \\
\hline \multicolumn{3}{|l|}{ USG } \\
\hline$\leq 1.029$ & $14 / 15$ & $2 / 4$ \\
\hline Isosthenuric (1.008-1.012) & $2 / 15$ & $0 / 4$ \\
\hline Hyposthenuric $(\leq 1.007)$ & $1 / 15$ & $0 / 4$ \\
\hline \multicolumn{3}{|l|}{ Hemoglobin (dipstick) } \\
\hline+ & $2 / 15$ & $1 / 4$ \\
\hline ++ & $3 / 15$ & $1 / 4$ \\
\hline+++ & $7 / 15$ & $0 / 4$ \\
\hline++++ & $2 / 15$ & $1 / 4$ \\
\hline \multicolumn{3}{|l|}{ Proteins (dipstick) } \\
\hline+ & $2 / 15$ & $4 / 4$ \\
\hline++ & $5 / 15$ & $0 / 4$ \\
\hline+++ & $7 / 15$ & $0 / 4$ \\
\hline \multicolumn{3}{|l|}{ Glucose (dipstick) } \\
\hline+ & $5 / 15$ & $0 / 4$ \\
\hline++ & $5 / 15$ & $0 / 4$ \\
\hline++++ & $2 / 15$ & $0 / 4$ \\
\hline \multicolumn{3}{|l|}{ Sediment examination } \\
\hline $\mathrm{RBC}>5 / \mathrm{hpf}$ & $10 / 15$ & $0 / 4$ \\
\hline $\mathrm{WBC}>3 / \mathrm{hpf}$ & $7 / 15$ & $0 / 4$ \\
\hline Tubular granular casts & $10 / 15$ & $0 / 4$ \\
\hline $\mathrm{UPC} \geq 0.5$ & $14 / 14$ & $2 / 4$ \\
\hline $\mathrm{UAC}>0.05$ & $14 / 14$ & $0 / 4$ \\
\hline \multicolumn{3}{|l|}{ SDS-PAGE } \\
\hline Pure tubular pattern & $1 / 13$ & $0 / 4$ \\
\hline \multicolumn{3}{|l|}{ Mixed pattern } \\
\hline$<23 \mathrm{kDa}$ & $6 / 13$ & $0 / 4$ \\
\hline$>23 \mathrm{kDa}$ & $6 / 13$ & $0 / 4$ \\
\hline Physiologic pattern & $0 / 13$ & $4 / 4$ \\
\hline
\end{tabular}

USG, urine specific gravity; RBC, red blood cells; hpf, high power field; WBC, white blood cells; UPC, urinary protein to creatinine ratio; UAC, urinary albumin to creatinine ratio; SDSPAGE, sodium dodecyl sulphate-polyacrylamide gel electrophoresis; kDa, kiloDalton.

survivors had DIC. DIC did not predict survival $(P=$ .126). The following variables were significantly higher in nonsurvivors compared with survivors: $\operatorname{cTnI}(P=$ $.009), \mathrm{CRP} / \mathrm{Hpt}(P=.012), \mathrm{UAC}(P=.013)$, and UPC $(P=.025)$. Serum albumin and total protein concentrations were significantly lower in nonsurvivors $(P=.005$ and $P=.03$, respectively). Other variables, such as ddimer, CRP, creatinine, and urea concentrations, were higher, but not significantly, in nonsurvivors $(P=.052$, $P=.08, P=.12$, and $P=.18$, respectively). Similarly, Hpt concentrations were lower, but not significantly, in nonsurvivors $(\mathrm{P}=.055)$. The frequency of urinary casts in survivors and nonsurvivors was not statistically different. Outcome was not affected by whether one or multiple organs were damaged. However, a poor outcome was significantly associated with cardiac involvement $(P=.033)$.
Only 1 asymptomatic dog showed alterations in hematologic, hemostatic, biochemical, or acute phase protein (APP) results. The dog in question had mild neutrophilic leukocytosis, mild thrombocytopenia, and an increase in serum CRP concentration $(1.57 \mathrm{mg} / \mathrm{dL}$; reference range, $0-0.5 \mathrm{mg} / \mathrm{dL}$ ). Urinalysis and SDSPAGE results were available for all asymptomatic dogs. Two of 4 dogs had mildly increased UPC (0.6 and 1.1, respectively), whereas the UAC was $<0.05$ in all asymptomatic dogs. Urinalysis results of asymptomatic dogs are reported in Table 4. Asymptomatic dogs were treated with doxycycline for 2 weeks.

\section{Discussion}

The "gold standard" for diagnosing leptospirosis still is the MAT ${ }^{2,46,10}$ even though, to the authors' knowledge, experimental and epidemiological studies aimed at establishing its predictive value currently are lacking in the veterinary medical literature. In Italy, the low prevalence of leptospirosis in dogs allows for interpretation of MAT titers of 1:100 (with the exception of serogroups Canicola and Icterohemorragiae) as indicative of infection. ${ }^{7,22}$ These thresholds differ from those adopted in North America and other European countries, where a higher single titer or a 4-fold increase in paired sera are recommended because of high endemicity. ${ }^{2-4,6,8,23}$

In this study, a presumptive diagnosis of leptospirosis was made based on clinical signs, clinicopathologic findings, and MAT serology. Of interest, nonsurvivors had lower MAT titers than survivors on admission. In 2/ 7 nonsurvivors, the presence of infection was supported either by a 4-fold increase in paired sera or by a titer of 1:3200 in a dog sharing the same household. The other 5 nonsurvivors died before reevaluation of titers could be performed. Because the length of time from the onset of clinical signs to admission was similar in survivors and nonsurvivors, the lower MAT titers detected in the latter could be explained by a shorter time between infection and onset of clinical signs in nonsurvivors. Consequently, the choice of MAT cutoff values potentially may bias the survival rate data in retrospective studies. Antigenic tests may improve the diagnosis of acute infections when increased MAT titer results are hampered by the acute nature of the disease or negative outcome.

Due to higher serologic reactivity to LIAS than to other serogroups, dogs in this study were considered infected by serovars belonging to the Australis serogroup. The paradoxical effect (ie, higher reactivity during the early phase of infection against a serogroup not actually responsible for the infection) purported to occur in dogs, ${ }^{4}$ and reported in people, ${ }^{10}$ was not seen in this study in those dogs in which paired sera were evaluated. Because several dogs died before paired sera could be assessed, it is possible that some dogs actually were infected with a different serogroup.

Because clinical signs were nonspecific, historic risk (eg, exposure to rural environments) raised diagnostic suspicion of leptospirosis. However, 2/20 dogs were not exposed to this risk factor. Therefore, the potential for 
contamination of domestic environments by underevaluated carriers such as hedgehogs should be investigated during historic data collection. ${ }^{22}$

SIRS and acute phase response were expected and commonly detected in this study as a likely consequence of the wide tissue tropism of leptospires ${ }^{1,9}$ and their demonstrated ability to induce cytokines release. ${ }^{9,11,13,14}$ In experimental studies of rats, guinea pigs, and human patients, both pro- and anti-inflammatory cytokines were increased in leptospiral infections. ${ }^{11,13,14}$ Nevertheless, the presence of SIRS at admission did not carry prognostic value in the dogs evaluated in this study. Because of the low number of animals evaluated, statistical analysis may have been inadequate to demonstrate a difference in outcome. Another possible explanation could be the low specificity of SIRS criteria for the severity of inflammation, as already evidenced in canine babesiosis. ${ }^{24}$

APPs increase in many infectious or inflammatory diseases of dogs, including leptospirosis. ${ }^{25-27}$ In general, the magnitude of APP increase reflects the extent of tissue damage, making measurement of APPs potentially helpful in determining outcome. ${ }^{25}$ In this study, an acute phase reaction was evidenced by at least 1 marker in all symptomatic dogs and in 1/4 asymptomatic dogs. Although concentrations of individual APPs at admission failed to predict outcome in the dogs studied here, the $\mathrm{CRP} / \mathrm{Hpt}$ ratio was significantly higher in nonsurvivors than in survivors $(P=.012)$. Because CRP increases more quickly and peaks earlier than Hpt in dogs (1-2 days versus 3-7 days, respectively), ${ }^{25}$ these results may reflect a shorter course of disease in nonsurvivors (a concept also supported by the significantly lower MAT titers in these dogs). An alternative explanation for the higher $\mathrm{CRP} / \mathrm{Hpt}$ ratios in nonsurvivors is that in vivo hemolysis may have decreased concentrations of $\mathrm{Hpt}$, which is rapidly cleared from the bloodstream after binding with free hemoglobin. ${ }^{25}$ However, this explanation is unlikely given that serum bilirubin concentrations and Hct results on admission did not differ significantly between survivors and nonsurvivors $(P=.89$ and $P=.186$, respectively).

There is a paucity of data about hemostasis in canine leptospirosis. When assessed with PT, aPTT, fibrinogen, and fibrin or fibrinogen degradation products (FDPs), DIC was reported to be uncommon in emerging leptospirosis. ${ }^{8}$ Conversely, coagulation abnormalities and DIC in particular were common findings in the dogs in this study regardless of whether or not clinical evidence of coagulopathy was present. The most frequent alteration was a reduction in AT activity that could have resulted from either increased consumption, protein-losing nephropathy, or both. Cook and Cowgill ${ }^{28}$ found a positive correlation between AT activity and serum albumin concentration in protein-losing glomerular disease in dogs. Therefore, the severe albuminuria evidenced in this study suggests that increased renal excretion may have exacerbated AT loss.

Decreased antithrombin activity, increased d-dimer concentrations, and thrombocytopenia were the most commonly fulfilled DIC criteria. Although different definitions exist, criteria for DIC in this study were more representative of subclinical DIC or a hypercoagulable state than of clinically overt DIC. More stringent criteria could have provided more specificity for the diagnosis of DIC but conversely would have reduced the sensitivity for detecting hypercoagulable states. Although d-dimer concentrations were higher in nonsurvivors, this difference was not significant $(P=.052)$. Neither the presence of DIC nor abnormalities in specific coagulation variables were significant predictors of outcome. These results may have been due in part to the fact that dogs with DIC were treated with aspirin, heparin, and fresh frozen plasma that may have improved their probability of survival.

Clinicopathologic data in dogs with LIAS infection identified multiple organ damage with consistent renal involvement and frequent involvement of muscle, heart, and liver. In the majority of symptomatic dogs, clinicopathologic evidence of nephropathy mirrored histologically apparent interstitial damage and was characterized by low USG, glucosuria, proteinuria, and casts formation. Similar findings were reported in previous studies in which infections caused by serogroups other than Australis were included. 1,2,6,8,29,30 Both UPC and UAC were significantly higher in nonsurvivors than in survivors and should be evaluated as outcome predictors in larger groups of dogs with leptospirosis.

In dogs with symptomatic LIAS infection, SDSPAGE most often identified a mixed pattern of proteinuria, consistent with the histologic evidence of both interstitial and glomerular lesions in these dogs. Similar findings previously were reported by Zaragoza and coworkers. ${ }^{18}$ Glomerular amyloidosis associated with nonamyloidotic tubulointerstitial inflammatory infiltrate was found in $1 \mathrm{dog}$. Renal amyloidosis has not previously been described as a direct consequence of leptospirosis, and secondary tubulointerstitial and glomerular lesions are reported to be absent or mild in dogs with renal amyloidosis. ${ }^{31}$ Therefore, the glomerular amyloidosis observed in $1 \mathrm{dog}$ in this study was most likely a preexistent lesion.

Serum concentrations of creatinine and urea did not differ significantly between survivors and nonsurvivors, although the latter group showed higher values. However, Adin and Cowgill ${ }^{2}$ did find a significant difference in serum creatinine and urea concentrations between survivors and nonsurvivors in their subset of dogs with leptospirosis $(\mathrm{n}=22)$ not treated by hemodialysis. These contrasting results may be related to differences in leptospiral pathogenicity, different MAT titer cutoffs used to include subjects, or different treatments. Moreover, the relatively low number of dogs included in this study may have been inadequate to demonstrate a significant difference in outcome. A remarkable success rate of $86 \%$ was reported by Adin and Cowgill ${ }^{2}$ for the subset of dogs with leptospirosis treated with hemodialysis, suggesting that hemodialysis represents the most effective therapeutic choice in anuric and oliguric patients. 
This study is the first to evaluate serum cTnI concentrations in dogs with leptospirosis, and results showed a high proportion of dogs with cardiac damage. This finding contrasts with the infrequency of overt arrhythmias detected on admission. Greenlee et $\mathrm{al}^{1}$ found epicarditis and endocarditis in dogs with experimentally induced leptospiral infections caused by serovar Pomona. However, because cTnI increase is caused by myocardial cell damage,,$^{32}$ epicarditis and endocarditis could not completely explain the results reported here. In humans, both myocarditis and thrombotic-hemorrhagic phenomena are implicated in the pathogenesis of leptospiral myocardial damage. ${ }^{9,10,33}$ The uremic syndrome is reported to be another possible cause of heart damage. ${ }^{33}$ However, the lack of correlation between urea and cTnI in the dogs in this study suggests that cardiac lesions associated with leptospirosis are likely due to direct myocyte damage rather than being secondary to azotemia. Serum cTnI concentrations were higher in nonsurvivors than in survivors $(P=$ .009 ), thus cTnI should be considered as a potential prognostic marker in LIAS infections.

Results of this study indicate that LIAS is frequently associated with systemic syndromes such as SIRS, DIC, and multiple organ damage. Although we did not find the presence of SIRS or DIC to be associated with outcome, this result may have been because of the relatively low number of animals studied. Hemostatic abnormalities, increased serum cTnI concentration, and marked albuminuria have not previously been well described in canine leptospirosis and warrant further investigation by larger prospective studies of leptospirosis caused by other serogroups as well as studies of other infectious diseases. Finally, serum albumin, total protein, cTnI, CRP/Hpt, UPC, and UAC all were found to be significantly associated with outcome in canine LIAS infections.

\section{Footnotes}

${ }^{a}$ Istituto Zooprofilattico Sperimentale della Lombardia e dell'Emilia Romagna, via Bianchi 1, Brescia, Italy. Istituto Zooprofilattico Sperimentale della Lombardia e dell'Emilia Romagna, via Fiorini 5, Bologna, Italy

${ }^{\mathrm{b}}$ Immulite Troponin I DPC, Medical System, Genova, Italy

${ }^{\mathrm{c}}$ Haptoglobin, Olympus Diagnostica, Milan, Italy

${ }^{\mathrm{d}}$ PHASE Canine C Reactive Protein ELISA, Tridelta Development Ltd, Celbio, Milan, Italy

${ }^{\mathrm{e}}$ Tina-quant d-dimer, Roche Diagnostici, Milan, Italy

${ }^{\mathrm{f}}$ Combur ${ }^{10}$ Test UX, Roche Diagnostici, Milan, Italy

${ }^{\mathrm{g}}$ Microalbumin, Olympus Diagnostica, Milan, Italy

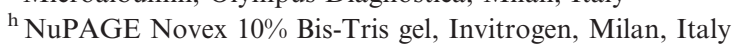

\section{Acknowledgments}

This study was supported by grants from Ricerca Fondamentale Orientata (RFO)-Università di Bologna (grants 2001-2002 and 2003-2004). The authors would like to thank the personnel of the "Istituto Zooprofilattico Sperimentale della Lombardia e dell'Emilia Romagna"a and especially Dr Maria Cristina Fontana for assistance in measuring and interpreting the MAT assay. The authors also are very grateful to Prof Cynthia M. Otto for helpful suggestions and critical reading of the article.

\section{References}

1. Greenlee JJ, Alt DP, Bolin CA, et al. Experimental canine leptospirosis caused by Leptospira interrogans serovars Pomona and Bratislava. Am J Vet Res 2005;66(10):1816-1822.

2. Adin CA, Cowgill LD. Treatment and outcome of dogs with leptospirosis: 36 cases (1990-1998). J Am Vet Med Assoc 2000; 216(3):371-375.

3. Boutilier P, Carr A, Schulman RL. Leptospirosis in dogs: A serologic survey and case series 1996 to 2001. Vet Ther 2003; 4(4):387-396.

4. Prescott JF, McEwen B, Taylor J, et al. Resurgence of leptospirosis in dogs in Ontario: Recent findings. Can Vet $\mathbf{J}$ 2002;43:955-961.

5. Bolin CA. Diagnosis of leptospirosis: A reemerging disease of companion animals. Semin Vet Med Surg Small Anim 1996; 11:166-171.

6. Birnbaum N, Barr SC, Center SA, et al. Naturally acquired leptospirosis in 36 dogs: Serological and clinicopathological features. J Small Anim Pract 1998;39:231-236.

7. Scanziani E, Origgi F, Giusti AM, et al. Serological survey of leptospiral infection in kenneled dogs in Italy. J Small Anim Pract 2002;43:154-157.

8. Rentko VT, Clark N, Ross LA, Schelling SH. Canine leptospirosis: A retrospective study of 17 cases. J Vet Int Med 1992;6:235-244.

9. Bharti AR, Nally JE, Ricaldi JN, et al. Leptospirosis: A zoonotic disease of global importance. Lancet 2003;3:757-771.

10. Levett PN. Leptospirosis. Clin Microbiol Rev 2001; 14(2):296-326.

11. Viriyakosol S, Matthias MA, Swancutt MA, et al. Toll-like receptor 4 protects against lethal Leptospira interrogans serovar Icterohaemorragiae infection and contributes to in vivo control of leptospiral burden. Infect Immun 2006;74(2):887-895.

12. Harkin KR, Roshto YM, Sullivan JT, et al. Comparison of polymerase chain reaction assay, bacteriologic culture, and serologic testing in assessment of prevalence of urinary shedding of leptospirosis in dogs. J Am Vet Med Assoc 2003;222(9): 1230-1233.

13. Yang CW, Wu MS, Pan MJ. Leptospirosis renal disease. Nephrol Dial Transplant 2001;16(5):73-77.

14. Diament D, Brunialti MK, Romero EC, et al. Peripheral blood mononuclear cell activation induced by Leptospira interrogans glycolipoprotein. Infect Immun 2002;70(4):1677-1683.

15. Johnson V, Gaynor A, Chan DL, Rozanski E. Multiple organ dysfunction syndrome in humans and dogs. J Vet Emerg Crit Care 2004;14(3):158-166.

16. Gentilini F, Dondi F, Mastrorilli C, et al. Validation of a human immunoturbidimetric assay to measure canine albumin in urine and cerebrospinal fluid. J Vet Diagn Inves 2005;17(2): 179-183.

17. Dondi F, Gentilini F, Giunti M, et al. Comparison of urinary total protein-creatinine ratio and albumin-creatinine ratio in evaluating proteinuria in dogs. Proceedings of the 13th European College of Veterinary Internal Medicine - Companion Animals (ECVIM-CA) Congress, Uppsala, Sweden, 4-6 September 2003. 
18. Zaragoza C, Barrera R, Centeno F, et al. Characterization of renal damage in canine leptospirosis by sodium dodecyl sulphate-polyacrylamide gel electrophoresis (SDS-PAGE) and Western Blotting of the urinary proteins. J Comp Pathol 2003;129(2-3):169-178.

19. Bazzi C, Petrini C, Rizza V, et al. Characterization of proteinuria in primary glomerulonephritides. SDS-PAGE patterns: Clinical significance and prognostic value of low molecular weight ("tubular") proteins. Am J Kidney Dis 1997;29: $27-35$.

20. Hauptman JG, Walshaw R, Olivier NB. Evaluation of the sensitivity and specificity of diagnostic criteria for sepsis in dogs. Vet Surg 1997;26:393-397.

21. Brady CA, Otto CM. Systemic inflammatory response syndrome, sepsis, and multiple organ dysfunction. Vet Clin North Am Small Anim Pract 2001;31(6):1147-1162.

22. Cerri D, Ebani VV, Fratini F, et al. Epidemiology of leptospirosis: Observations on serological data obtained by a "diagnostic laboratory for leptospirosis" from 1995 to 2001. Microbiologica 2003;26:383-389.

23. Steger-Lieb A, Gerber B, Nicolet J, Gaschen F. Eine alte Krankheit mit neuem Gesicht: Die Hundeleptospirose verliert nicht an Aktualität. Schweiz Arch Tierheilkd 1999;141:499-507.

24. Welzl C, Leisewitz AL, Jacobson LS, et al. Systemic inflammatory response syndrome and multiple-organ damage/ dysfunction in complicated canine babesiosis. J S Afr Vet Assoc 2001;72(3):158-162.

25. Cerón JJ, Eckersall PD, Martínez-Subiela S. Acute phase proteins in dogs and cats: Current knowledge and future perspectives. Vet Clin Pathol 2005;34(2):85-99.

26. Yamamoto S, Shida T, Miyaji H, et al. Changes in serum Creactive protein levels in dogs with various disorders and surgical traumas. Vet Res Commun 1993;17:85-93.

27. Caspi D, Snel WJJ, Batt RM, et al. C-reactive protein in dogs. Am J Vet Res 1987;48:919-921.

28. Cook AK, Cowgill LD. Clinical and pathological features of protein-losing glomerular disease in the dog: A review of 137 cases (1985-1992). J Am Anim Hosp Assoc 1996;32:313-322.

29. Wohl JS. Canine leptospirosis. Compend Contin Educ Pract Vet 1996;18(11):1215-1241.

30. Harkin KR, Gartrell CL. Canine leptospirosis in New Jersey and Michigan: 17 cases (1990-1995). J Am Anim Hosp Assoc 1996;32:495-501.

31. DiBartola SP, Tarr MJ, Parker AT, et al. Clinicopathologic findings in dogs with renal amyloidosis: 59 cases (1976-1986). J Am Vet Med Assoc 1989;195(3):358-364.

32. Lobetti R, Dvir E, Pearson J. Cardiac Troponins in canine babesiosis. J Vet Intern Med 2002;16:63-68.

33. De Zoysa JR. Cardiac troponins and renal disease. Nephrology 2004;9(2):83-88. 